Ann. Zootech., I980, 29 (3), 305-3I5.

\title{
Paramètres biochimiques et digestibilités dans le duodénum chez le poney recevant un même aliment complet granulé ou semi expansé
}

\author{
R. WOLTER, Andrée DURIX, D. GOUY, J.-C. LETOURNEAU \\ Monique CARCELEN et Jacqueline GOUY \\ avec la collaboration technique de A. BRUNy et A. VII, I,ARD \\ Laboratoire de Nutrition et d'Alimentation \\ École Nationale Vétérinaire de Lyon, I.N.R.A. \\ Marcy l'Étoile, 69260 Charbonnières-les-Bains (France)
}

\begin{abstract}
Résumé
Un même aliment complet ( 12,6 p. Ioo de protéines brutes et 12,3 p. 1oo de cellulose) est présenté sous forme granulée ou semi-expansée (seule la partie céréalière subit un traitement hydrothermique), à 2 poneys porteurs d'une canule au niveau du duodénum. Sur ces animaux rationnés à $2 \mathrm{~kg} /$ animal /jour distribués en 2 repas d'une $\mathrm{I} / 2$ heure, les prélèvements sont effectués toutes les 2 heures après le repas pour l'estimation des digestibilités partielles et toutes les heures pour le contrôle des paramètres biochimiques.

Dans les conditions d'un régime restreint et fractionné l'expansion-extrusion de la partie céréalière améliore la digestibilité duođénale de manière significative pour la matière sèche et les matières azotées, et presque significative pour l'amidon.

Ces résultats, qui reflètent la digestion stomacale, font ressortir que si la fraction protéique est encore peu attaquée (CUD $a=-$ I et $9 \mathrm{p}$. roo), la partie amylacée est déjà fortement dégrađée (CUD $a=58$ p. Ioo et $69 \mathrm{p}$. I oo) sous les actions fermentaires. Une proportion importante d'acide propionique apparaît 2 à 4 heures après le repas alors que 1'acide lactique ne se forme qu'après 5 à 6 heures; ces 2 métabolites sont plus abondants avec l'aliment semi-expansé, et, parallèlement, le $\mathrm{pH}$ tend à être plus bas.
\end{abstract}

\section{I. - Introduction}

L'influence de la technologie de l'aliment sur la digestion chez les équidés a été abordée par la comparaison de trois présentations d'un même aliment complet: granulé, expansé et semi-expansé. Il n'est pas apparu de différence significative des digestibilités totales. (WolTer, Durix, LE'TOURneau, I976). Pour mieux préciser le site et la vitesse de digestion de la ration, des digestibilités partielles 
ont été déterminées, d'une part grâce à la récolte du contenu des différents segments du tube digestif chez des animaux abattus (Gouy 1976, ChaAbouni I977), et d'autre part, en mettant à profit des poneys porteurs de fistules permanentes (WOLTER et al., I978). Ainsi, sur 3 poneys munis d'une fistule caecale, nous n'avons pas constaté d'influence sensible de la présentation de 1'aliment complet (granulé, expansé ou semi-expansé) sur la digestibilité totale, la digestibilité atteinte au niveau du caecum et sur le métabolisme intracaecal. Cependant, des essais pratiques sur chevaux de sport ont montré que la valeur énergétique de l'aliment semble nettement améliorée par l'expansion (WOLTER et al., r977).

Dès lors, il était utile d'évaluer la digestion dans les sites antérieurs du tube digestif. A cet effet, sur 2 poneys porteurs de fistule au niveau du duodénum, nous avons comparé l'évolution de la composition du contenu duodénal pour un même aliment complet granulé ou semi-expansé. Les résultats présentés concernent :

- la composition de la matière sèche du contenu duodénal et la digestibilité partielle en amont du duodénum;

- les paramètres biochimiques du fluide duodénal : $\mathrm{pH}$, taux d'acides gras volatils, d'acide lactique, d'ammoniac qui traduisent l'intensité des dégradations microbiennes intervenant dans l'estomac.

\section{II. - Matériel et méthodes}

\section{r. - Animaux}

Les essais ont été conduits sur 2 poneys placés en cage à métabolisme et munis d'une canule au niveau du duodénum. Cette canule en plastique souple (obtenue par moulage de plastisol vinylique) est constituée par un tube cylindrique de $8 \mathrm{~cm}$ de long et de $I, 7 \mathrm{~cm}$ de diamètre fixé sur une partie demi-cylindrique, qui s'applique contre la paroi interne de l'intestin.

La mise en place de cette canule duodénale est comparable à celle des canules caecales (WOLTER et al., I 978). Eille se déroule en 2 temps : au cours d'une première intervention chirurgicale, la paroi du duodénum est extériorisée à environ $20 \mathrm{~cm}$ du pylore, en aval du canal pancréatique; elle est solidarisée avec le péritoine, les muscles et la peau, de manière à susciter une solide adhérence périphérique; une semaine plus tard, la paroi duodénale est incisée et la canule est implantée.

\section{2. - Aliments}

Une même formule (tabl. I) est granulée ou semi-expansée, c'est-à-dire que dans ce dernier cas la partie céréalière est soumise au procédé d'expansion (WOL,TER et al., I 976, I978). I a composition centésimale de chaque présentation est rapportée dans le tableau $I$.

Ces aliments sont distribués à raison de $2 \mathrm{~kg} / \mathrm{Al} / \mathrm{jour}$, en 2 fois à $8 \mathrm{~h}$ et $\mathrm{I} 7 \mathrm{~h}$. Au cours de la période d'adaptation de 3 semaines, les animaux ont été entraînés à consommer leur ration en I heure puis en une demi heure. 


\begin{tabular}{|c|c|c|}
\hline & \multicolumn{2}{|c|}{ p. roo } \\
\hline $\begin{array}{l}\text { Orge (Barley) } \\
\text { Avoine (Oats) } \\
\text { Maïs (Maize) } \\
\text { Issues de blé (Milling by-products). } \\
\text { Farine de luzerne (Lucerne meal). } \\
\text { C.M.V. (Minerals and vitamins) }\end{array}$ & & \\
\hline & $\begin{array}{c}\text { Granulé } \\
(\text { Pelleted })\end{array}$ & $\begin{array}{c}\text { Semi-expansé } \\
\text { (Semi-extruded) }\end{array}$ \\
\hline $\begin{array}{l}{[\text { Matière sèche }(\text { Dry matter })]} \\
\text { Matières azotées }(\mathrm{N} \times 6,25)(\text { Crude protein }) \text { p. Ioo MS } \\
\text { Lignocellulose }(\text { Van Soest ADF }) \\
\text { Cellulose vraie }(A D F \text {-lignine }) \\
\text { Lignine (Van Soest-Lignin) }\end{array}$ & $\begin{array}{r}89,64 \\
12,20 \\
\mathrm{I} 6,28 \\
\mathrm{I} 2,3 \mathrm{I} \\
3,97 \\
28,40 \\
\mathrm{II}, \mathrm{Or}\end{array}$ & $\begin{array}{r}89,43 \\
12,80 \\
16,44 \\
12,38 \\
4,06 \\
30,70 \\
10,07\end{array}$ \\
\hline
\end{tabular}

\section{3. - Prélèvements}

\section{a) Composition des contenus duodénaux et digestibilités partielles}

Les échantillons sont constitués par le contenu duodénal qui s'écoulespontanément de la canule et que l'on collecte dans des bécliers refroidis pendant des périodes successives de 2 heures; à la suite du repas du matin, d'abord entre o et 2 heures puis entre 2 et 4 heures, enfin entre 4 et 6 heures.

Au delà de cette durée, il ne s'écoule pratiquement plus de particules solides.

On admet que la composition moyenne de ces 3 échantillons est un reflet de la composition moyenne des digesta.

Sur chaque animal on réalise trois séries de prélèvements à 3 jours d'intervalle, d'abord au mois de mai, puis au mois de septembre. Les résultats sont alors les moyennes des 6 valeurs obtenues sur deux animaux.

La teneur en matière sèche de chaque prélèvement est calculée après dessication à l'étuve à $80^{\circ} \mathrm{C}$ pendant 72 heures. Les Coefficients d'Utilisation Digestive (CUD) partiels ont été évalués en utilisant la lignine comme marqueur, celle-ci s'étant révélée bien adaptée à la détermination des CUD partiels et totaux. lors de nos précédents essais avec les aliments complets (WolTER et al., I978).

\section{b) Contrôles biochimiques}

Les prélèvements sont effectués à l'aide d'une sonde plongeant par l'intermédiaire de la canule dans la lumière intestinale. Étant donné les faibles quantités 
prélevées (ro $\mathrm{ml}$ de liquide duodénal sur I goutte de merthiolate à I p. Ioo) ils sont renouvelés toutes les heures pendant les 8 heures consécutives à la distribution du repas.

Après contrôle immédiat du $\mathrm{pH}$, ces échantillons sont préparés en vue des dosages suivants :

- ammoniac, par dilution au I/4 dans 1'acide trichloracétique à 2,5 p. Ioo;

- acides gras volatils par congélation directe après centrifugation;

- acide lactique, par dilution au I/3 par l'acide perchlorique à $0,6 \mathrm{~N}$.

\section{4. - Techniques d'analyses}

Les aliments ainsi que les prélèvements du contenu duodénal sont soumis aux analyses de leurs constituants bruts afin d'en déterminer la composition et d'évaluer les coefficients d'utilisation digestive :

- matières azotées totales $(\mathrm{N} \times 6,25)$, par la méthode de Kjeldahl;

- glucides membranaires selon la technique de Van Soest (I963) : lignocellulose $(\mathrm{ADF})$, lignine et cellulose (ADF-lignine);

- amidon après hydrolyse enzymatique à l'amyloglucosidase et dosage du glucose (ChARLIER et al., I974).

L,es contrôles biochimiques sont effectués par les méthođes déjà relatées (WOLTER et al., I978) :

- le $\mathrm{pH}$ au pHmètre immédiatement après le prélèvement;

- les acides gras volatils par chromatographie en phase gazeuse après acidification par $\mathrm{H}_{3} \mathrm{PO}_{4}$ de l'échantillon congelé;

- l'acide lactique par voie enzymatique à la lacticodéshydrogénase (réactif bio-Mérieux);

- l'ammoniac par la réaction de Berthelot sur autoanalyseut Technicon (MrcheL, I97I).

\section{III. - Résultats}

\section{I. - Évolution de la matière sèche et de ses constituants}

Le tableau 2 récapitule l'évolution du taux de matière sèche et de ses différents constituants aux cours des 6 heures consécutives aux repas du matin.

- L'évacuation gastrique de la matière sèche, qui apparaît élevée au cours des 2 premières heures qui suivent le repas, décroît ensuite rapidement quelle que soit la présentation de l'aliment.

- Les pourcentages de matières azotées dans la matière sèche, peu différents de ceux de 1'aliment, croissent très légèrement entre le I $^{\text {er }}$ et le dernier prélèvement (ils sont légèrement plus fort avec l'expansé) pour lequel la digestibilité est elle-même significativement plus haute $(\mathrm{P}<0,05)$. 
离

\&

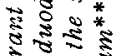

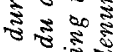

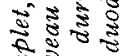

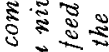

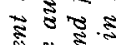

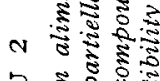

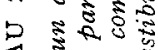

闩

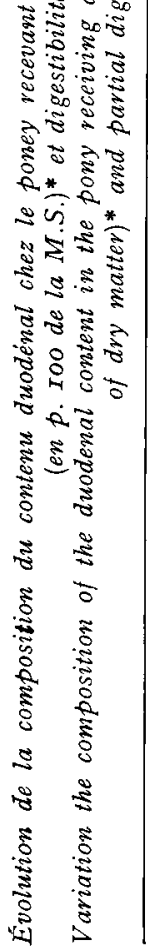

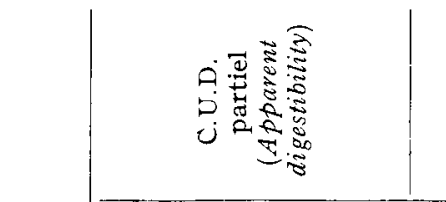

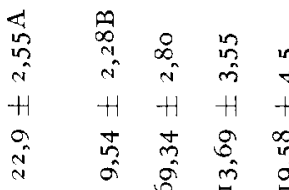

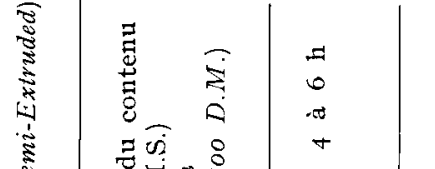

曹造:

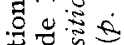

㟧

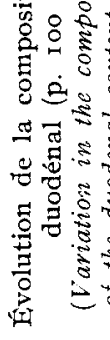

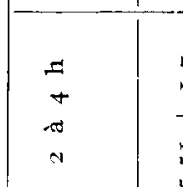

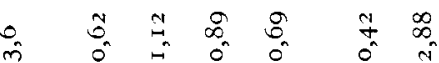

$\mathrm{H} H+H \quad H \quad H$

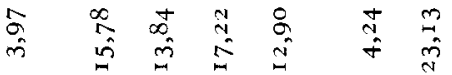

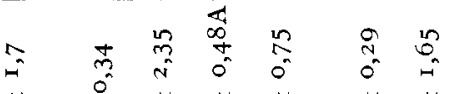

$\mathrm{H} H \mathrm{H} H$ H H

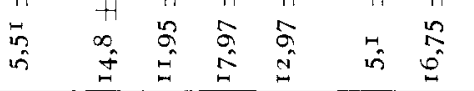

事

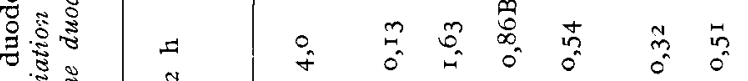

H H H H H H H

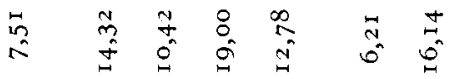

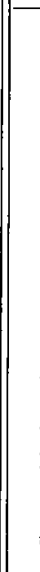

\begin{tabular}{|c|c|c|c|c|c|c|c|}
\hline \multicolumn{2}{|c|}{ 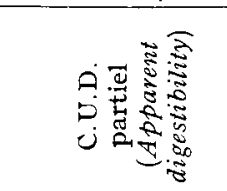 } & $\begin{array}{l}\text { to } \\
\text { on } \\
\text { in } \\
+1 \\
8 \\
\text { : } \\
\Rightarrow\end{array}$ & 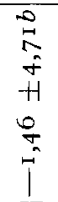 & $\begin{array}{l}0 \\
\text { s } \\
H \\
0 \\
0 \\
\infty \\
i n\end{array}$ & 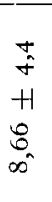 & $\begin{array}{l}\hat{0} \\
0 \\
+1 \\
0 \\
0 \\
\vdots \\
=\end{array}$ & 1 \\
\hline \multirow{3}{*}{ 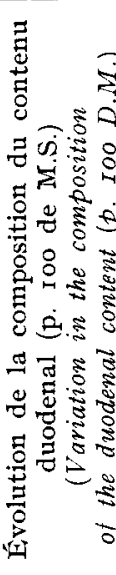 } & $\begin{array}{l}\Rightarrow \\
0 \\
\Leftrightarrow \\
+\end{array}$ & $\begin{array}{l}0 \\
\infty \\
0 \\
+1 \\
b \\
\vdots \\
\sigma\end{array}$ & $\begin{array}{l}0 \\
8 \\
0 \\
H \\
0 \\
i n \\
0 \\
1\end{array}$ & $\begin{array}{l}0 \\
0 \\
\dot{f} \\
H \\
0 \\
0 \\
m \\
n\end{array}$ & 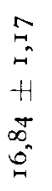 & $\begin{array}{l}9 \\
0 \\
0 \\
H \\
+ \\
+ \\
\text { a }\end{array}$ & $\begin{array}{l}\hat{b} \\
0 \\
+1 \\
0 \\
0 \\
\text { oे }\end{array}$ \\
\hline & $\begin{array}{l}\Rightarrow \\
+ \\
+\infty\end{array}$ & \begin{tabular}{l}
0 \\
in \\
$-H$ \\
\multirow{2}{*}{}
\end{tabular} & $\begin{array}{l}m \\
3 \\
0 \\
H \\
H \\
0 \\
\vdots \\
\vdots \\
j\end{array}$ & $\begin{array}{l}n \\
+ \\
H \\
H \\
+ \\
m \\
m \\
\end{array}$ & 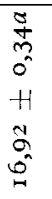 & 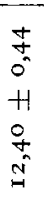 & $\begin{array}{l}\frac{17}{0} \\
0 \\
+1 \\
5 \\
\frac{2}{7}\end{array}$ \\
\hline & $\begin{array}{c}\pi \\
0 \\
0\end{array}$ & 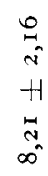 & $\begin{array}{l}0 \\
0 \\
0 \\
0 \\
+1 \\
0 \\
0 \\
0 \\
0\end{array}$ & 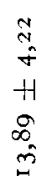 & 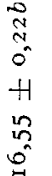 & $\begin{array}{l}y \\
0 \\
0 \\
+4 \\
0 \\
0 \\
=\end{array}$ & $\begin{array}{l}8 \\
0 \\
0 \\
11 \\
8 \\
0 \\
f\end{array}$ \\
\hline
\end{tabular}

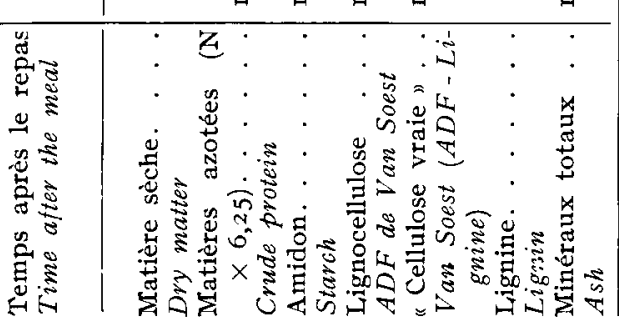

$\dot{H}$
$\dot{0}$
0
$V$
$\dot{1}$

$\infty$

$\rightarrow$

in

E

4

$\stackrel{0}{2}$

8

畩

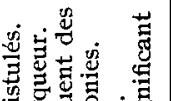

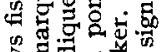

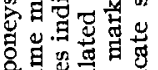
६ 웜․․ 战豆。

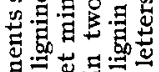

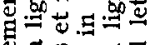
월

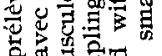

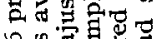

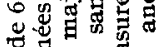

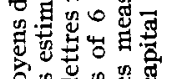

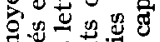

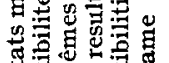

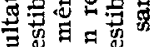

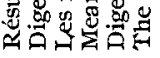


- La proportion d'amidon dans cette matière sèche reste relativement constante dans les 6 heures qui suivent le repas, mais ne représente que la moitié de la teneur dans l'aliment, démontrant ainsi une disparition précoce de ce constituant, dès l'estomac. En outre, ces taux d'amidon sont plus faibles avec l'aliment semi-expansé dont la digestibilité amylacée est plus élevée $(69,3$ p. roo) que la forme granulée $(58,0 \mathrm{p}$. I00) la différence étant presque significative $(\mathrm{P}<0,05)$.

- Les teneurs en lignocellulose et “cellulose vraie » varient peu avec l'ordre des prélèvements et demeurent légèrement supérieures avec l'aliment semiexpansé.

- La lignine se concentre quelque peu dans les digesta, par suite de la disparition des autres constituants.

- Le taux des matières minérales totales, s'élève au cours du temps, particulièrement dans les 4 à 6 heures après le repas, pour atteindre 2 fois la proportion dans l'aliment. Leur digestibilité, fortement négative, est identique pour les 2 présentations.

\section{2. - Evolution de la composition biochimique du fluide duodénal}

Le $p H$ : proche de 7 juste après le repas (fig. I), s'abaisse progressivement au cours de la journée jusqu'à des valeurs égales ou inférieures à 6 ; il n'apparaît pas de différence significative en fonction du type d'aliment.
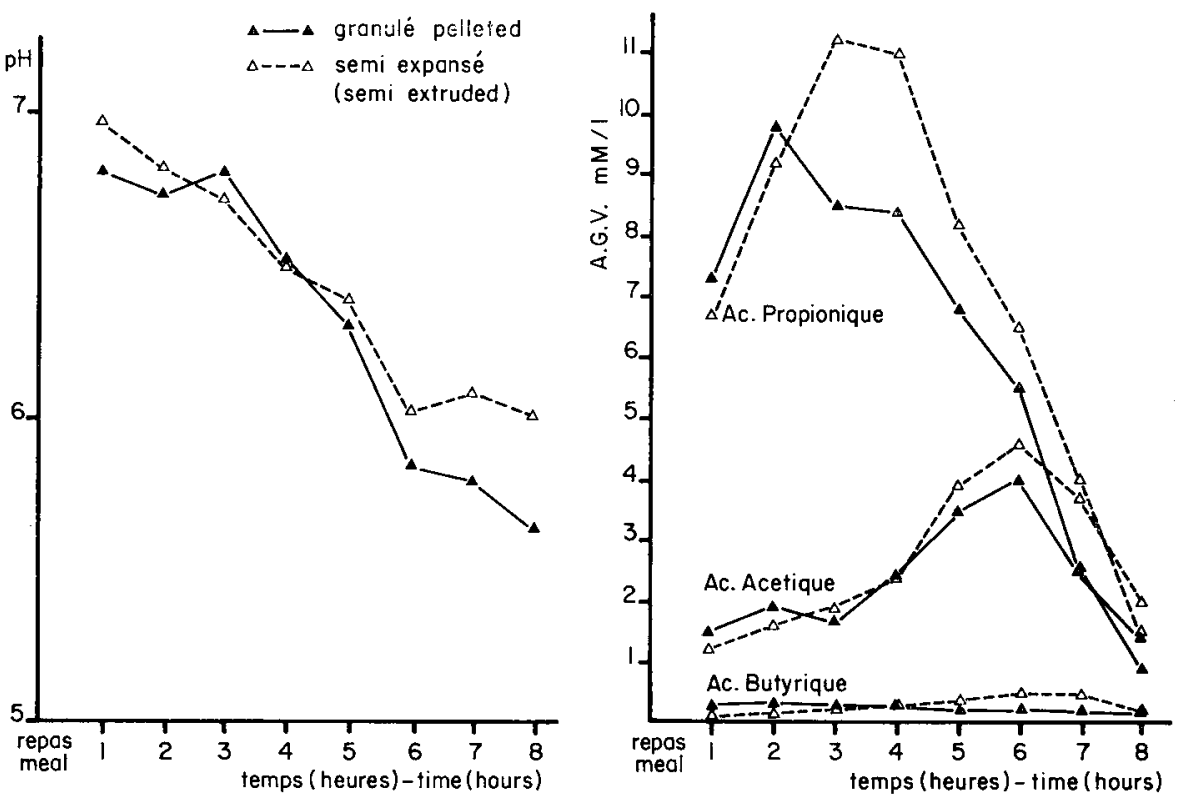

FIG. I. - Evolution du $p H$ du fuide duodenal.

pH changes in the duodenat fuid.

FIG. 2. - Cinétique des taux d'acides gras volatils dans le duodenum (mM/1). Variation in VFA concentration in duodenal fuid. 
Les acides gras volatils: l'acide propionique relativement abondant passe par un maximum 2 à 4 heures après le repas (9 à $\mathrm{I} 2 \mathrm{mM} / 1$ ), pour diminuer ensuite (fig. 2), alors qu'augmente l'acide acétique dont les concentrations demeurent faibles ( 2 à $4 \mathrm{mM} / 1$ ); l'acide butyrique n'apparaît qu'à l'état de traces.

L'acide lactique: les taux en sont élevés (fig. 3) et atteignent 6 à $8 \mathrm{mM} / 1 \mathrm{en}$ moyenne 6 heures après le repas ; les différences entre les 2 présentations sont alors significatives.

L'ammoniac: les teneurs en sont assez hautes : de $50 \mathrm{mg} / 1$, elles croissent jusqu'à I3o mg/1 3 à 4 heures après le repas (fig. 4).
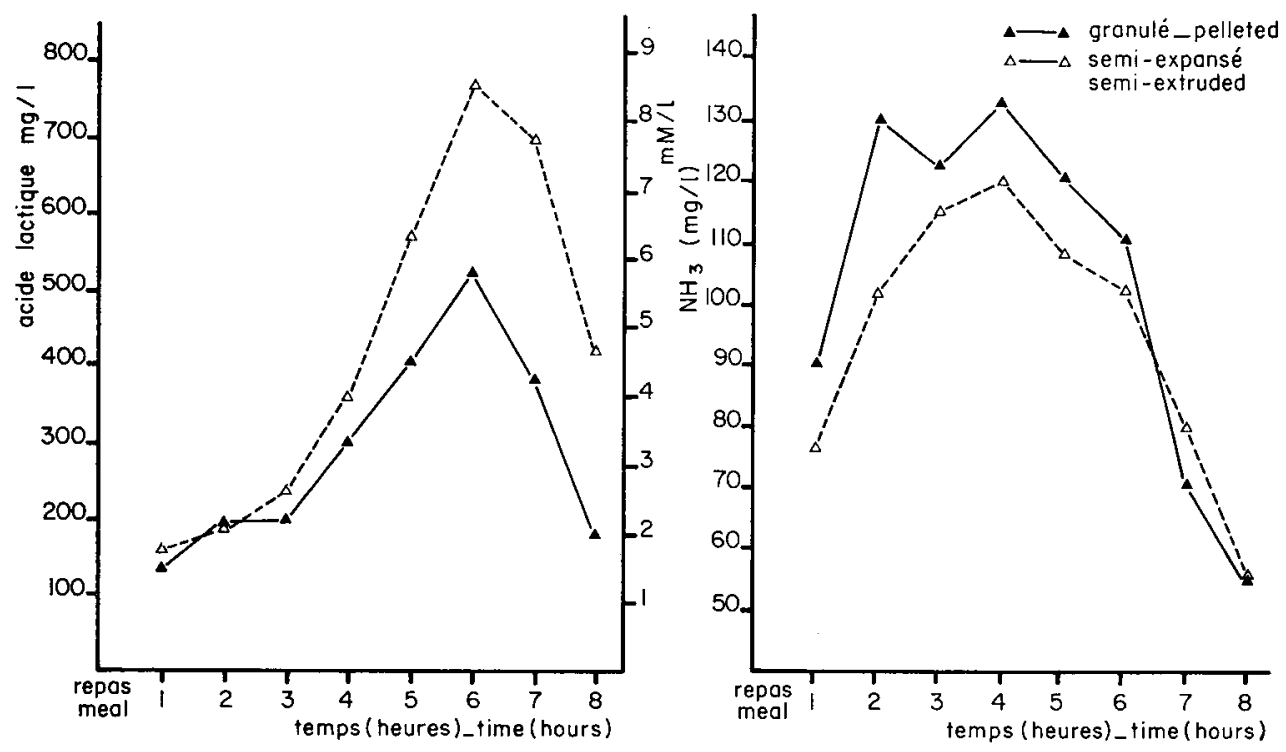

FIG. 3. - Évolution du taux d'acide lactique dans le fluide duodénal (mg/1 et mM/1). Variation of lactic acid concentration in the duodenal fluid.

FIG. 4. - Évolution du taux d'ammoniac du fuide duodenal (mg/1).

Variation of ammonia concentration in the duodenal fluid.

\section{IV. - Discussion}

\section{I. - Importance de la digestion dans l'estomac}

Etant donné la position de la canule $(20 \mathrm{~cm}$ en aval du pylore, donc en aval du canal pancréatique), les effluents recueillis traduisent essentiellement l'influence de la digestion gastrique. Celle-ci s'exerce pendant un temps court puisque la vidange stomacale est très active au cours des premières heures qui suivent le repas, à l'image de ce qui se déroule chez d'autres monogastriques tels que le pore (CUBER et LAPLACE, I979). De ce fait, la digestibilité gastrique de la matière sèche reste faible (tab1. 2), mais pas négative comme cela a déjà été démontré 
avec des rations associant fourrages et céréales en différentes proportions (HrNTZ et al., I97I) ou avec des aliments complets (ChAa Bouni, I977). Par comparaison, des résultats très comparables ont par ailleurs été obtenus chez le porc (KEYs et DE BARTHE, I $974 a$ et I974b).

La digestibilité apparente des matières azotées demeure également presque nulle à la sortie de l'estomac. Il en est de même chez le lapin recevant un aliment complet (WoL'TER, Nouwakpo et Durix, I980). Cette digestibilité apparente peut même devenir nettement négative sous l'influence de fortes sécrétions digestives, stomacales et duodénales, ainsi que cela a été vérifié chez le poney dans notre laboratoire (El, Aouni, I980), et chez le porc (Keys et DE BARTHE, I974b).

Au contraire, 1'amidon est assez largement digéré ( 58 à 69 p. Ioo) dès l'estomac, vraisemblablement pour une part sous l'influence de la microflore gastrique. Déjà nous avions constaté chez le poney que $64 \mathrm{p}$. Ioo de l'amidon disparaît dans l'estomac au cours des 3 heures qui suivent le repas, et, chez le cheval, que la digestibilité de 1'amidon atteint 86 p. Ioo au niveau du premier tiers de l'intestin grêle. Ces résultats recoupent bien ceux auxquels parviennent chez le porc KEYS et $\mathrm{DE}$ BARTHE, I974b) qui situent entre 45 et $75 \mathrm{p}$. Ioo la digestibilité duodénale de 1'amidon, selon la nature de celui-ci.

Parallèlement, les glucides membranaires apparaissent dégradés de façon non négligeable (I I et I 9 p. Ioo), ce qui confirmerait la relative intensité des fermentations microbiennes au niveau de l'estomac, tout au moins dans les conditions de nos essais : petits repas, aliment divisé, à faible taux de cellulose et conditionné.

La digestibilité assez fortement négative des minéraux totaux traduit l'abondance des apports endogènes, déjà mis en évidence chez des équidés abattus (Gouy, I976). Elle peut aussi rendre compte partiellement de la modeste digestibilité apparente des matières sèches malgré la digestion précoce de l'amidon qui représente $30 \mathrm{p}$. Ioo de la ration.

\section{2. - Contrôles biochimiques et orientation des fermentations}

Le pH intraduodénal diminue au cours du temps postprandial (fig. I), alors qu'augmentent les concentrations en acides organiques. En particulier, le taux des acides gras volatils croît jusque $\mathbf{I} 2 \mathrm{mM} / 14$ heures après le repas (fig. 2 ) et s'amenuise ensuite au fur et à mesure de la réduction du substrat et de la progression de l'absorption digestive; ainsi, il apparaît bien intermédiaire entre ceux antérieurement trouvés dans l'estomac $(5 \mathrm{I} \mathrm{mM} / 1)$ et dans l'intestin grêle $(6$, Io $\mathrm{mM} / \mathrm{l})$ chez des poneys recevant un régime comparable (NouWa kPo, I979); par contre, il reste très inférieur à ceux constatés dans le caecum : Ioo à I6o mM/1 (WoLTER et al., I978, Nouwakpo, I 979). Cette production gastrique d'acides gras volatils confirme l'action fermentaire, déjà admise par ALEXANDER et FARRIES (I963), et particulièrement propice à la libération d'acide propionique (fig. 2), compte tenu des conditions de $\mathrm{pH}$ et de la richesse de l'aliment en amidon. Au contraire, avec un régime cellulosique, le rapport acide acétique/acide propionique se trouverait inversé (ARG-ENZIO, SouTHWORTH et STEVENS, 1974).

A la faveur de l'abaissement renforcé du $\mathrm{pH}$ gastrique à laquelle elle contribue directement, en plus de l'effet propre de la sécrétion chlorhydrique, l'activité fermentaire se poursuit jusqu'à la formation d'acide lactique, qui est plus tardive que la libération des acides gras volatils (fig. 3).

Il en résulte que la teneur duodénale en acide lactique est sensiblement plus élevée (6 et $8 \mathrm{mM} / 1)$ que dans le caecum ( $3 \mathrm{mM} / 1)$ de poneys fistulés (WoL,TER 
et $a l, \mathrm{r} 978)$; plus précisément, i1 a été montré que la concentration en acide lactique, qui est maximale dans l'estomac et la partie initiale de l'intestin grêle, devient à peu près nulle dans les segments postérieurs du tube digestif (ARG-ENZIo et al., I974; Chadbouni, i 977; Wolter et ChaAbouni, I979). De même, chez le porc, 1'acide lactique est bien représenté jusqu'à la fin de l'intestin grêle (ÉTIENNE, I97I), alors que chez le lapin, il semble absent y compris dans l'estomac (NouWA KPO, I979).

Au total, il ressort que la dégradation microbienne des glucides dans l'estomac des équidés n'est pas négligeable et peut amoindrir la valeur énergétique potentielle des aliments facilement fermentescibles, à base d'amidon et surtout de sucres, sinon exposer à des risques d'acidose digestive.

Parallèlement, il semble se dérouler dans l'estomac, voire aussi dans l'intestin grêle, une notable altération microbienne des protéines alimentaires puisque le taux d'ammoniac atteint dans le duodénum I 20 à $130 \mathrm{mg} / 1$ dans les 2 heures qui suivent le repas, alors que dans des conditions similaires, nous n'avons mis en évidence au niveau du caecum de poneys fistulés que des concentrations de 50 à $80 \mathrm{mg} / 1$ (WOLTER et al., r978); toutefois, ces valeurs restent bien inférieures à celles rapportées à partir d'équidés abattus (CHA A.BOUNI, I977) chez lesquels des dégradations post-mortem ont $\mathrm{pu}$ fortement les exagérer.

\section{3. - Influence du mode de présentation de l'aliment}

Par rapport à la simple mise en granulés de l'aliment, l'expansion-extrusion de la partie céréalière améliore la digestibilité duodénale, de manière significative pour la matière sèche, ainsi que les protéines, et presque significative pour l'amidon. Parallèlement, il accroît la production gastrique d'acide propionique et d'acide lactique. Il stimule donc la digestion précoce de l'amidon, par voie fermentaire, dans l'estomac, voire par voie enzymatique dans le premier tiers du jejuno-iléon (ChaAbount, I977), bien que dans les conditions de nos précédents essais, nous n'ayons pas constaté de différence quant aux digestibilités caecale (WoLTER et al., I976), ou totale (WoLTER et Gouy, I976).

Par comparaison, chez le porc le traitement hydrothermique des céréales augmente quelque peu la digestibilité de la matière sèche, des substances organiques et des protéines (Borgida, I975; Aumaitre et Dumont, r975). De façon plus nette chez le ruminant, il accélère les fermentations ruminales, en privilégiant la libération d'acide propionique favorable au rendement énergétique chez l'animal en engraissement; rapidement même, il renforce les risques d'acidose lactique.

Ces mêmes risques d'origine gastrique pourraient éventuellement apparaître chez les équidés lors de l'ingestion brutale ou exagérée de glucides très facilement fermentescibles. Cependant, il semble que chez les équidés les accidents majeurs d'acidose lactique dérivent du caecum lors d'indigestion amylacée.

\section{Conclusion}

Cette étude, qui demanderait à être complétée par des essais portant sur la nature de l'amidon distribué, confirme l'attaque précoce de la fraction amylacée de l'aliment chez le poney, vraisemblablement par fermentation microbienne dans 
l'estomac. L'apport de glucides fermentescibles doit donc être assez strictement mesuré et fractionné afin d'éviter tout risque d'acidose consécutive à une ingestion trop brutale d'amidon surtout si celui-ci a subi un traitement hydrothermique, exposant alors à des troubles digestifs ou métaboliques.

Accepté pour publication en aồt Ig8o.

\section{Summary}

\section{Measurement of digestibility and biochemical parameters in the dwodenum of ponies fed a diet of ordinary or semi-extruded pellets}

Two ponies with permanent fistulas of the duodenum were fed ordinary of semi-extruded pellets (semi-extruded $=$ moist heat treatment of the cereal fraction only) containing I 2.6 p. IOo protein and $12.3 \mathrm{p}$. Ioo crude fibre (Table $\mathrm{I}$ ).

The animals received only $2 \mathrm{~kg}$ feed /animal / day in 2 meals of half an hour each. Samplings were made every 2 hours after the meal for digestibility determinations, and every hour for control of the biochemical parameters.

In these conditions of severely restricted and fractionated feeding the two physical forms of the feed led to a significant improvement of the duodenal digestibility coefficients of dry matter, crude protein and starch (Table II) or as regards metabolism in duodenal fluid (measured by $\mathrm{pH}$, ammonia, volatile fatty acids, and lactic acid concentrations) (fig. $\mathrm{I}, 2,3$ and 4 ).

Crude protein digestibility is low ( $-\mathrm{I}$ and $+9 \mathrm{p}$. Ioo) while starch is well degraded ( $5^{8}$ and 69 p. 10o). There are high levels of propionic acid (between 2 and 4 hours after the meal) (fig. 2) and of lactic acid (5-6 hours after the meal) (fig. 2) and of lactic acid (5-6 hours after the meal) (fig. 3). These two acid levels are higher and the $\mathrm{pH}$ is lower with the semi-extruded diet.

\section{Références bibliographiques}

AlEXANDER F., FARrifs Elizabeth, I963. Production and fermentation of lactate by bacteria in the alimentary canal of the horse and pig. J. Com. Path., 73, I-8.

ARGENZIO R. A., SOUTH WORTH M., STFVENS C. E., I974. Site of organic acid production and absorption in the gastro intestinal tract. Ann. J. Physiol., 225, $1043^{-1050 .}$

Aumaitre A., Henky Y., Mercier C., Ivorec-Szylit O., Thivend P., i972. Étude préliminaire de l'influence d'un traitement hydrothermique sur la valeur alimentaire du blé et du maïs. Ann. Zootech. 21, I33-137.

Aumaitre A., Dumond R., i 975 . Utilisation des céréales dans les régimes de sevrage précoce du porcelet $=$ comparaison de l'orge et du blé et efficacité de quelques traitements hydrothermiques. Journées Recherche porcine en France, I5I-I6o. I.N.R.A., I.T.P. éd. Paris.

BoRGIDA L. P., I975. Floconnage et expansion de l'orge et du mais. I. - Étude technologique. II. - Digestion du maïs floconné chez le porc en croissance. Journées de Recherche porcine en France, 99-I04. I.N.R.A., I.T.P. éd., Paris.

Chanbouni A., i 977. Nouvelle étude de la digestion de l'anidon chez les équidés par analyse du contenu du tube digestif après abattage. Thèse de Doctorat Vétérinaire, Lyon.

Charlier C., Van Efenamin C., Canart B., Mondant A., Lambót O, Bienfait J. M., I974. Méthode de dosage semi-automatique de l'amidon et du glucose dans les aliments du bétail. Ann. Méd. Vét., 118, 28 [-294.

CUBER J. C., LAPI,ACE J. P., r979. Fívacuation gastrique de la matière sèche d'un régime semipurifié à base d'amidon de maïs chez le porc. Ann. Biol. anim. Bioch. Biophys., 19, 899-905.

Durand Michelle, Ben Ameur M., Viroben G., i 974 . Influence d'un traitement hydrothermique de l'orge sur la digestion des matières azotées dans le rumen du mouton : études au niveau du rumen et de l'abomassum. Ann. Biol. anim. Bioch. Biophys., 14, 167-192. 
ÉTIENNE M., i97I. Prođuction d'acides gras à courte chaîne au cours de la digestion chez le porc. Ann. Biol. anim. Bioch. Biophys., 11, 341 .

Gouy D., I976. Une étude de la digestion chez les équidés par analyse du contenu intestinal après abbatage. Thèse de Doctorat Vétérinaive, Lyon.

Hintz H. F., Hogue D. E., Walker E. F., LowE J. E. Schriver, I97I. Apparent digestion in the digestive tract of ponies fed diets with varying roughage-grain ratio. J. Anim. Sci, 32,245 .

KEYS J. E., DE BARTHE J. V., I974a. Cellulose and hemicellulose in the stomach, small intestine and large intestine of swine. $J$. anim. sci., 36, 53-56.

KEYS J. F,, DE BARTHE J. V., I974 b. Site and extent of carbohydrate dry matter, energy and protein digestion and the rate of passage of grain diets in swine. J. anim. sci., 36, 57-62.

LAWRENCE J. T. L., I972, A review of some effects on health and performance of variations in the physical form of the diet of the growing pig. III. - Heat treatment and cubing. Veterinary. Record., 61, I08-III.

Miches M. C., I97I. Analyse quantitative de quelques substatice azotées et glucidiques en milieu biologique. Essai de rationnalisation. Thèse de Doctorat d'Université, Clermont-Ferrand.

Nouwakpo F., I 979. Une étude expérimentale de la digestion chez le lapin comparaison avec le poney. Thèse de doctorat d'ingénieur, Dijon.

REMESY C., DEMIGNE C., 1976. Partition and absorption of volatile fatty acids in the alimentary canal of the rat. Ann. Rech, Vet., 7, 39-55.

VAN SOEST J. P., 1963. Use of detergents in the analysis of fibrous feed II. A rapid method of the determination of fiber and lignin. J. A.O.A.C., 46, 829-835.

Wolter R, Andrée Durix, Leitourneau J. C., I974. Influence du mode de présentation du fourrage sur la vitesse de transit digestif chez le poney. Ann. Zootech., 23, 293-30o.

Wolter R., Andrée Durix, Letourneau J. C., i975. Influence du mode de présentation du fourrage sur la digestibilité chez le poney. Ann. Zootech., 24, 237-242.

Wolter R., Andrée Durix, Lestourneau J. C., i976. Influence du mode de présentation d'un aliment complet sur la vitesse du transit digestif et la digestibilité chez le poney. Ann. Zootech., 25, I 8 I-I 88 .

WOLTER R., Gouy D., I976. Étude expérimentale de la digestion chez les équidés par analyse du contenu intestinal après abattage. Rev. Méd. Vét., 127, 1723-1 736.

Wolter R., Meunier B., de Faucompret R., Andrée Durix, Jacqueline Landreau, 1977. Essai d'un aliment complet, granulé ou expansé, en comparaison avec le régime traditionnel chez des chevaux de sport. Rev. Méd. Vét., 128, 7 I-8I.

Worter R., Gouy D., Andrée Durix, Letourneau J. C., I978. Digestibilité et activité biochimique intracaecale chez le poney recevant un même aliment complet, présenté sous forme granulée, expansée ou semi-expansée. Ann. Zootech. 27, 47-6o.

Wolter R., ChaAbGUNi A., I979. Étude de la digestion de l'amidon chez le cheval par analyse du contenu digestif après abattage. Rev. Med. Vét., 130, I 345-I 357 .

Wor Ter R., Nouwakpo F., Atidrée Durix, 1980. Étude comparative de la digestion d'un aliment complet chez le poney et le lapin. Reproduction, Nutrition, Développement 20, I 723-1 730. 